\title{
The involvement of bottom-up saliency processing in endogenous inhibition of return
}

\author{
David Henderickx • Kathleen Maetens • Eric Soetens
}

Published online: 26 October 2011

(C) Psychonomic Society, Inc. 2011

\begin{abstract}
Participants are faster at detecting a visual target when it appears at a cued, as compared with an uncued, location. In general, a reversal of this cost-benefit pattern is observed after exogenous cuing when the cue-target interval exceeds approximately $250 \mathrm{~ms}$ (inhibition of return [IOR]), and not after endogenous cuing. We suggest that, usually, no IOR is found with endogenous cues because no bottom-up saliency-based orienting processes are claimed. Therefore, we developed an endogenous feature-based split-cue task to allow for endogenous saliency-based orienting. IOR was observed in the saliency-driven endogenous cuing condition, and not in the control condition that prevented saliency-based orienting. These results suggest that usage of saliency-based orienting processes in either endogenous or exogenous orienting warrants the appearance of IOR.
\end{abstract}

Keywords Inhibition of return - Attention: Selective

\section{Introduction}

Which elements in the processes underlying inhibition of return (IOR) can account for both its appearance in most exogenous cuing studies and its absence in most endogenous cuing tasks? In the present study, we examined the possibility of IOR being grounded on bottom-up saliencydriven orienting processes. Whereas exogenous cues act

D. Henderickx $(\bowtie) \cdot K$. Maetens $\cdot$ E. Soetens

Department of Experimental and Applied Psychology,

Vrije Universiteit Brussel,

Pleinlaan 2,

1050 Brussels, Belgium

e-mail: David.Henderickx@vub.ac.be upon stimulus saliency, which is associated with low-level stimulus processing, endogenous cues are mostly associated with higher-level processing, involving the task set in working memory. Accordingly, the difference in processing level may account for the variance in the appearance of IOR. To investigate the hypothesis that IOR is linked to mechanisms of low-level saliency processing, we designed a series of experiments with a split-cue technique in order to create endogenous cuing conditions with and without low-level saliency processing.

\section{Attentional facilitation and inhibition}

Because of restraints in perceptual processing capacity, attentional mechanisms have to direct the available resources of the visual system toward the portion of the visual field from which to extract information (Wolfe, 1994). This attentional selection may be controlled by the stimulus (stimulus driven) or by the observer (goal driven), which Posner (1980) referred to as exogenous and endogenous attention, respectively.

Posner's (1980) cuing task is one of the prevailing paradigms used for studying visual attention. In this kind of task, the pattern of attentional costs and benefits is measured by the manipulation of a visual warning signal. The warning signal, or cue, preceding the onset of the target stimulus, indicates the location of the upcoming target with a certain probability. With short cue-target intervals (CTIs), participants are faster at detecting the visual target when its position is validly cued (cuing benefits) than when it is invalidly cued (cuing costs; Posner \& Cohen, 1984). This facilitation effect has been demonstrated in numerous studies and is thought to reflect speeded perceptual processing of the attended target (Jonides, 1981; Müller \& Rabbitt, 1989). 
When attention is exogenously directed to a location due to attentional capture of an abrupt onset cue (attentional capture; Remington, Johnston, \& Yantis, 1992), the regular facilitation of processing at the cued location lasts only a brief period of time (approximately $250 \mathrm{~ms}$ ) after cue onset. When CTI exceeds this period, not only does the facilitatory benefit after valid cuing vanish, but also an additional cuing cost sets in. That is, validly cued targets are detected more slowly than uncued and invalidly cued targets. This reversal of the cost-benefit pattern has been termed IOR, to reflect the idea that attention is inhibited from returning to a previously attended location (Posner \& Cohen, 1984; Posner, Rafal, Choate, \& Vaughn, 1985).

To study exogenous attention, uninformative cues are usually implemented in the Posner paradigm, since exogenous attentional selection is considered to be independent of our intentions (e.g., Jonides \& Yantis, 1988; Yantis \& Jonides, 1984). In this way, the target appears with the same likelihood at the cued location than at any other location. Accordingly, participants have no obvious grounds for sustaining attention at the cued location. Moreover, participants are told to ignore the cue and to respond only to the onset of the target. Given the noninformative nature, it would be strategically appropriate to redistribute attentional resources over the visual field to optimally detect the target onset if no target appears shortly after the attentional capture of the cue. It is assumed that participants "zoom out" of the attended location and reorient attention to a central location that is equidistant from each potential target location. Consequently, the lack of spatial information, along with the automatism of orienting to the exogenous cue, could explain why cuing benefits are observed only at short CTIs. Various explanations have been proposed regarding the appearance of IOR after longer CTIs. On the one hand, the attentional account (also called the reorienting hypothesis; Lupiáñez et al., 2004) suggests that IOR reflects a bias of attention toward novel locations and against returning to previously attended objects and locations (Klein, 1988; Posner \& Cohen, 1984). Inhibited objects or locations no longer compete for attentional selection, so that the orientation toward uninspected locations is encouraged and, conversely, reinspection of locations where no targets have been found is discouraged (Posner \& Cohen, 1984). On the other hand, the oculomotor account posits that IOR is rather a consequence of suppressing the motor system to make a saccade toward a previously peripherally cued location (Taylor \& Klein, 1998). Both behavioral and brain studies have accumulated evidence that both attentional and motor systems may be involved in the generation of IOR (Danziger, Ward, Owen, \& Rafal, 2002; Dorris, Klein, Everling, \& Munoz, 2002; Kingstone \& Pratt, 1999; Mayer, Seidenberg, Dorflinger, \& Rao, 2004; Sapir, Soroker, Berger, \& Henik, 1999).
Generally, it is agreed that IOR places inhibitory tags on objects or locations that recently have been inspected.

IOR clearly increases the efficiency of visual search. For example, Klein (1988) presented a target on half of the trials at either a previously inspected location or a previously uninspected location. Responses were slowed for detecting the target at previously inspected locations in a conjunction task, which required allocation of attention. In a feature task, where no focused attention was required, no reaction time (RT) lengthening was found. This may be taken as evidence that IOR is needed to prevent the attentional focus from returning to previously inspected locations (see also Müller \& von Mühlenen, 2000). Moreover, when searching complex scenes, Klein and MacInnes (1999) found that saccadic RT was longest to a target presented at a previously searched location and that there was a decrease in saccadic RT as the distance from this location increased, which is what would be expected if IOR helps search. Finally, Theeuwes and Godijn (2004) demonstrated that irrelevant distractors compete less when presented at inhibited locations than when presented at noninhibited locations.

Both the facilitation and inhibition (IOR) of stimulus processing at previously attended locations have been widely studied in the last decade. In general, research has shown that IOR is a very robust phenomenon that reflects an important general principle of information processing.

\section{Endogenous orienting and oculomotor processes}

In contrast to exogenous attention, endogenous attention is voluntary. That is, under endogenous orienting conditions, participants do have grounds for sustaining attention at the cued location. Accordingly, endogenous facilitatory cuing effects reach a maximum at stimulus onset asynchronies longer than $300 \mathrm{~ms}$, remain relatively stable over time, and usually do not display IOR (e.g., Jonides \& Irwin, 1981; Müller \& Findlay, 1988; Rafal \& Henik, 1994). However, when the endogenous cue triggers the oculomotor system to prepare or execute a saccade, the inhibition (IOR) may surprisingly reappear in the cost-benefit pattern (Posner et al., 1985; Rafal, Calabresi, Brennan, \& Sciolto, 1989).

Rafal et al. (1989) suggested that saccade programming is a necessary condition for IOR to occur. In their study, IOR was examined after exogenous (peripheral) and endogenous (central) cuing and with varying instructions concerning the usage of the cue. The results revealed IOR in all conditions after exogenous cuing. With endogenous cuing, however, IOR was observed only when participants had executed or were instructed to prepare a saccade. When participants had to keep their eyes centrally fixed and make a covert attention shift, no IOR was found. These results are consistent with evidence from neuropsychological studies 
that the superior colliculus (SC), a midbrain oculomotor structure involved with both attention and oculomotor processes (Sparks \& Hartwitch-Young, 1989), is somehow involved in the generation of IOR (e.g., Dorris et al., 2002; Posner et al., 1985; Sapir, Hayes, Henik, Danziger, \& Rafal, 2004; Sapir et al., 1999).

A single-cell recording study by Dorris et al. (2002) provided evidence that IOR is caused by inhibition preceding oculomotor programming in the spatial saccade map (most likely in the SC). In this study, neural activity of cells in the SC was recorded while monkeys performed an IOR task that required a saccade to a peripheral target. During the CTI, neural activity was higher at the cued location than at the uncued location. Accordingly, there was no evidence that the neural activity at the cued location was actively inhibited. However, at the moment the target appeared, the increase of neural activity of the SC cells was greater when the target appeared at an uncued location than when it appeared at a cued location. This suggests that the inhibition causing IOR is not applied in the saccade map but, rather, within a system that provides input to the saccade map (see also Godijn \& Theeuwes, 2004; Theeuwes, Van der Stigchel, \& Olivers, 2006). That is, IOR seems to rely on low-level visual processes that underlie oculomotor programming and that also guide exogenous (i.e., stimulus-driven) attention shifts. Therefore, the conspicuous appearance of IOR when an endogenous cue triggers the oculomotor system to prepare or execute a saccade could possibly be explained by usage of these lowlevel orienting processes.

\section{Feature-based attention and working memory}

In the present study, we will focus on these bottom-up visual processes that feed the oculomotor and the exogenous orienting system. To examine their role in the occurrence of IOR, we developed a design that manipulates the use of these low-level bottom-up processes in endogenously guided attentional selection. Particularly, we are interested in the course of the cost-benefit pattern after endogenous cuing through top-down modulation of bottomup selection processes. Top-down knowledge may cause the bottom-up selection process to operate on particular features of the visual input. As a consequence, locations possessing that particular feature will potentially benefit further processing (feature-based attentional selection; e.g., Desimone \& Duncan, 1995; Folk \& Remington, 1998, 1999, 2006; Treisman \& Gelade, 1980).

Recent studies have provided evidence that the content of working memory (WM) interacts with selective attention (Olivers, Meijer, \& Theeuwes, 2006; Sala, \& Courtney, 2009; Soto, Heinke, Humphreys, \& Blanco, 2005). That is, $\mathrm{WM}$ enables the visual system to selectively maintain a limited amount of currently relevant information in an active state, to influence perceptual processing top-down. The interaction can be either facilitative or inhibitory and happens in a very task-specific manner (see also Woodman \& Luck, 2007). For example, when the shape of a sample object is kept in WM, a shape-matching distractor affects subsequent search, but a color-matching distractor does not. Similarly, when the color of the sample object is stored in WM, the matching color interferes, but the matching shape does not (Olivers et al., 2006). These results are consistent with the biased competition model of Desimone and Duncan (1995), who suggested that the visual system is biased for the information that is currently needed for the task at hand. The model predicts that when an observer's task is to select a specific target object from an upcoming stimulus display, consisting of multiple competing objects, the representation of the target will be preactivated, reflecting the activity of the content of WM. As a result, when the stimulus display is presented, the target is favored in the competition for attentional selection. The idea that top-down processes may influence automatic, stimulus-triggered processes is not new and is reflected in Folk's contingent-capture theory (Folk \& Remington, 1998, 1999, 2006; Folk, Remington, \& Johnston, 1992; Folk, Remington, \& Wright, 1994). The theory focuses on the concept of attentional set. When an observer has an attentional set for an object or for certain features of objects, these particular (object) features will capture attention when presented in the perceptual field.

An alternative explanation of top-down modulation of lowlevel selective attention is found in Wolfe's guided search theory. According to this theory, a preattentive parallel featureencoding process, resulting in several feature maps, is assumed to serve as a bottom-up component. Through interaction with top-down goal-driven processes, activation in these feature maps guides the focus of attention in a serial fashion (Cave \& Wolfe, 1990; Wolfe, 1994, 2007). That is, current behavioral goals or task demands, and the subjective state of the observer, determine to what extent different feature contrast maps contribute to a final activation map and, consequently, the focus of attention (Navalpakkam \& Itti, 2005; Wolfe, 1994, 2007; cf. the saliency map in Itti \& Koch, 2000, 2001 and Koch \& Ullman, 1985, and the conspicuity map in Henderickx, Maetens, Geerinck, \& Soetens, 2009).

The present study: Rationale

Several studies support the idea that the use of low-level visual processes underlying oculomotor programming and exogenous (stimulus-driven) orienting is possibly a prerequisite for the appearance of IOR (Dorris et al., 2002; Godijn \& Theeuwes, 2004; Theeuwes et al., 2006; the present study). Also, if volitional eye movements occur through high-level top-down modulation of the low-level visual 
system that underlies reflexive (i.e., stimulus-driven) saccadic eye movements (Clark, 1999), it would explain the appearance of IOR after endogenous cuing when eye movements are triggered (see, e.g., the study of Klein \& MacInnes, 1999). If this assumption is valid, IOR will always appear after exogenous cuing, whether or not the oculomotor system is triggered, and after endogenous cueing only when the lowlevel saliency map is top-down modulated and, hence, when eye movement programming is triggered. Accordingly, these predictions agree to a large extent with the results and suggestion of Rafal et al. (1989) and the majority of cuing patterns after exogenous cuing (e.g., Posner \& Cohen, 1984).

To test the suggestion that the use of saliency-based orienting processes is a prerequisite for IOR, we set up a series of endogenous cuing experiments in which attention was guided to a well-defined location in visual space. We developed a feature-based cuing paradigm with a split cue. Here, a central instruction was presented to direct attention to the cued location by using a central color cue and two peripheral location cues. This central color cue was presented at fixation in one of three possible colors. The peripheral cues appeared left and right from fixation, at the two possible target locations. They mutually differed in color, with one peripheral cue being in the same color as the central cue and the opposite peripheral cue in one of the two other colors. The color of the central color cue instructed participants as to which peripheral color they needed to orient - that is, to the peripheral location that was cued with the same color as the central cue. In this way, participants endogenously oriented attention to the peripheral cue with the same color as the central cue.

The two peripheral colored cues were presented simultaneously on both sides of fixation, so that no exogenous attention capture was elicited to one particular side. When the target appeared at the location of the peripheral cue with the same color as the central cue, the trial was considered valid. When the target appeared at the peripheral location with the differently colored cue, the trial was considered invalid. In all experiments within this study, cues were nonpredictive concerning the target location. Nonetheless, participants were urged to comply with the endogenous cuing instructions. During the experiments, validity checks were implemented for the shortest CTI to control for the use of the central cue and to give online feedback when performance deteriorated. With this split-cuing design, we were able to realize endogenous cuing, with attentional orientation being determined by the modulation of WM content on low-level visual processes.

Important in this split-cuing task was the onset time between the central and peripheral cues. When participants were given sufficient time to store (buffer) the color of the central cue in WM, we expected a top-down process of WM that would elicit an activation bias in a low-level saliency map. Subsequently, saliency-based orienting to the peripheral location with the same color as the central cue could be triggered (Experiments 1, 5, and 6). In an endogenous control condition, central and peripheral cues were presented simultaneously (Experiments 2 and 4), or the central cue was presented after the offset of the peripheral cues (Experiment 3), to prevent the formation of an attentional set. That is, we expected participants to orient attention by intentionally comparing peripheral cues with the central cue (or by perceptual grouping of equal colors; addressed in Experiment 3), since an a priori activation bias of the central cue color was missing. If the usage of bottom-up saliency information is a prerequisite for IOR, an inverse cost-benefit pattern (IOR) with endogenous cuing conditions would be expected only when there was sufficient time to process the central cue before arrival of the peripheral cue.

\section{Experiment 1: Attentional set on each trial (short CTI range)}

In a first experiment, we attempted to modulate the lowlevel attentional maps by means of an endogenous split cue with sufficient time between the central and peripheral cues. After central fixation offset, activation of a particular feature in WM was accomplished by presenting a central color cue. The central cue was presented to participants as an instruction as to which peripheral location they needed to orient. After the central cue, the peripheral cue followed, where participants had to orient to the cue with the same color as the central cue. In this first experiment, the attentional set was determined for each trial. That is, on each trial, the color of the central cue was randomly drawn out of three colors. Sufficient time between the onset of the central cue and the onset of the peripheral cues allowed participants to preset attention for the central cue color. We expected to find a reversed cost-benefit pattern when the CTI exceeded about $250 \mathrm{~ms}$.

\section{Method}

Participants Twenty university students (University of Brussels) between 18 and 24 years of age were recruited to participate for course credits. All had normal or corrected-to-normal vision and no color blindness. All participants were naive with respect to the purpose of the study, and none of them had previous experience with cuing tasks.

Apparatus and stimuli For all experiments, stimuli and instructions were presented on a Dell M782p 17-in. CRT monitor with a refresh rate of $60 \mathrm{~Hz}$. The display was 
driven by the RAGE 128 PRO II internal graphics card in a Dell Dimension $8300(2.40 \mathrm{GHz})$ PIV IBM-compatible personal computer controlled in real time by the experiment application suite E-Prime version 1.1 (Schneider, Eschman, \& Zuccolotto, 2002). All displays were viewed binocularly at a viewing distance of approximately $50 \mathrm{~cm}$ in a laboratory test box, which was dimly illuminated. Responses were registered by means of pressing keyboard buttons.

Procedure Before participants started the actual experiment, they were orally introduced to the split-cue task, accompanied by a visual example of a trial. The meaning of covert orienting was explained to the participants; that is, they were advised "to search the matching peripheral cue from the corner of the eyes, without actually moving the eyes."

Each trial consisted of the subsequent presentation of six types of displays: fixation, central color cue, peripheral location cue, CTI, target, and feedback display (see Fig. 1). The fixation consisted of a white point in the middle of a gray rectangular field centered on a black background. The gray rectangular field was vertically and horizontally centered on the screen, measuring $34^{\circ} 22^{\prime}$ horizontal and $8^{\circ} 53^{\prime}$ vertical visual angle. Duration of the fixation display was $800 \mathrm{~ms}$, after which the central color cue was presented.

The central color cue was a square, vertically and horizontally centered on the screen, measuring $2^{\circ} 86^{\prime}$ horizontal and $2^{\circ} 86^{\prime}$ vertical visual angle, and colored in one of three colors: red, blue, or yellow. The duration of the color cue was $300 \mathrm{~ms}$, after which the peripheral location cues were presented. The peripheral cues had the same size as the central color cue and were presented left and right from fixation immediately after offset of the central color cue. Visual angle between the centers of the left and right peripheral location cues measured $22^{\circ} 78^{\prime}$. The duration of the peripheral location cues was $100 \mathrm{~ms}$. The combination of cues (split cue) was noninformative; that is, $50 \%$ of the cues were valid. Although participants could not predict the location of the upcoming target, they were urged to comply with the endogenous cuing instructions. Participants were told that they could lose course credits ${ }^{1}$ if they regularly disobeyed the cue instructions or task performance was inferior. During the experiment, a real-time control procedure continuously calculated and compared mean RTs for valid and invalid cues within the shortest CTI condition to control for the use of the central cue. When mean RTs

\footnotetext{
${ }^{1}$ None of the participants lost their course credits, since the performance check was meant only to stimulate performance.
}

indicated disobedience of the cue instructions, after each 60 trials, a 10-s visual pop-up appeared that reminded the participant about the proper use of the cues.

The CTI display was a duplicate of the fixation screen and had a random duration of $100,200,300,400$, or $500 \mathrm{~ms}$. The CTI reflected the time starting from the offset of the peripheral cue until the onset of the target. After the CTI, a gray square appeared as the target and remained on screen until participants responded to its location (left or right) by pressing the corresponding left or right arrow key. The target remained on screen for a maximum duration of 3,000 ms. After a correct answer, RT was shown for $1,000 \mathrm{~ms}$. When the response was incorrect, an error message was presented in red.

Design After the instructions, a practice block of 40 trials was presented. When accuracy was above $85 \%$, an experimental block of 240 trials was started. When participants did not reach the accuracy level, instructions and practice trials were repeated. After each series of 60 trials, a 10-s break was inserted. Cue validity and CTI were manipulated randomly within subjects. The order of combinations of central cue color and peripheral distractor color was random and equalized over all trials. Targets were randomly and equally distributed over both locations for all conditions. Critical dependent variables were RT and accuracy.

\section{Results}

The results of Experiment 1 are presented in Fig. 2. Each participant's data for the experimental block were used to calculate mean RTs and accuracies for valid and invalid spatial cues for each CTI. Only RTs for correctly responded trials were used. Both RT and accuracy data were subjected to a repeated measures analysis of variance (ANOVA), with CTI and cue validity as variables.

The overall effect of CTI was significant, $F(4,76)=83,89$, $p<.001, \eta_{p}^{2}=.815$. Participants responded faster as CTI increased. Over all CTI conditions, a marginally significant cuing effect appeared when the valid and invalid cuing conditions were compared, $F(1,19)=4.10, p<.10, \eta_{p}^{2}=$ .178. Importantly, the interaction between CTI and cue validity was significant, $F(4,76)=13.64, p<.001, \eta_{p}^{2}=$ .418 .

Analyses of each CTI condition separately indicated significant effects between the valid and the invalid cuing conditions in the 100-ms condition (37 ms), $F(1,19)=21.26$, $p<.001, \eta_{p}^{2}=.528$, and in the $200-\mathrm{ms}$ condition (27 ms), $F$ $(1,19)=8.13, p<.01, \eta_{p}^{2}=.300$. In the 300- and 400-ms CTI conditions, no effect of validity was observed, $F(1,19)=$ 
Fig. 1 Course of a trial for the split-cue Experiment 1 with attentional set. The cue-target onset interval between the split cue (location cues after a central cue) and target onset was manipulated

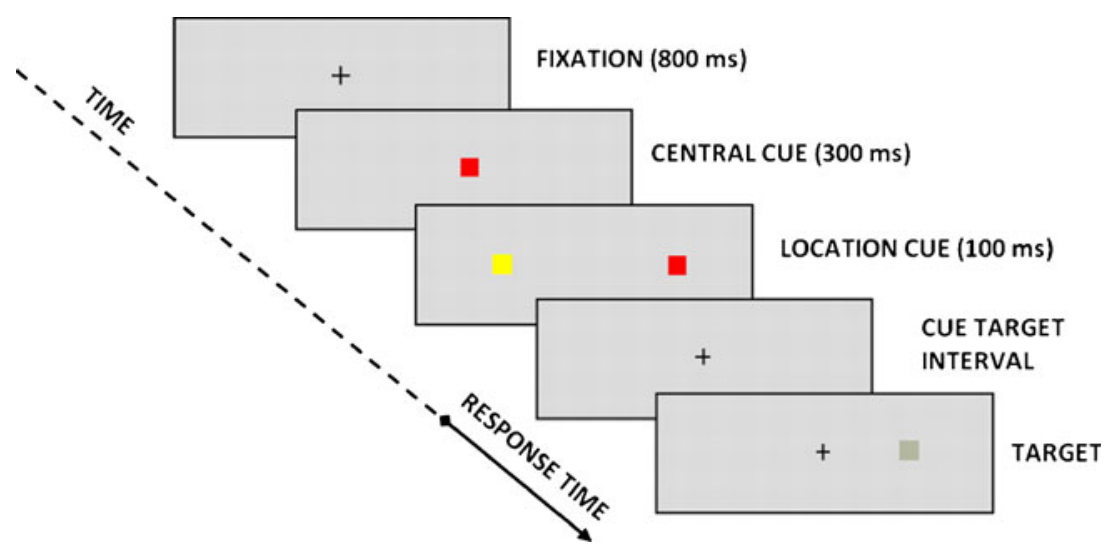

2.90, n.s., $\eta_{p}^{2}=.132$, and $F(1,19)=0.30$, n.s., $\eta_{p}^{2}=.015$, respectively. In the 500-ms CTI condition, a reversed cuing effect was observed (-19 ms) - that is, longer RTs for valid than for invalid cues, $F(1,19)=8.36, p<.01, \eta_{p}^{2}=.300$.

The RT results showed that IOR can be observed for the 500-ms CTI condition. In the shorter CTI conditions (i.e., with 100- and 200-ms intervals), a normal cuing pattern was found. It is noteworthy that the IOR phenomenon appeared after a 500-ms CTI, while in the traditional exogenous IOR experiments, the reversal already appeared after about a 250-ms CTI.

The accuracy analysis did not reveal any significant effects. In all conditions, participants approximated the maximum performance level.

\section{Experiment 2: No attentional set (simultaneous split cue; short CTI range)}

In a second experiment, we changed the experimental design in order to prevent participants from using low-level saliency processing in endogenous orienting. The time

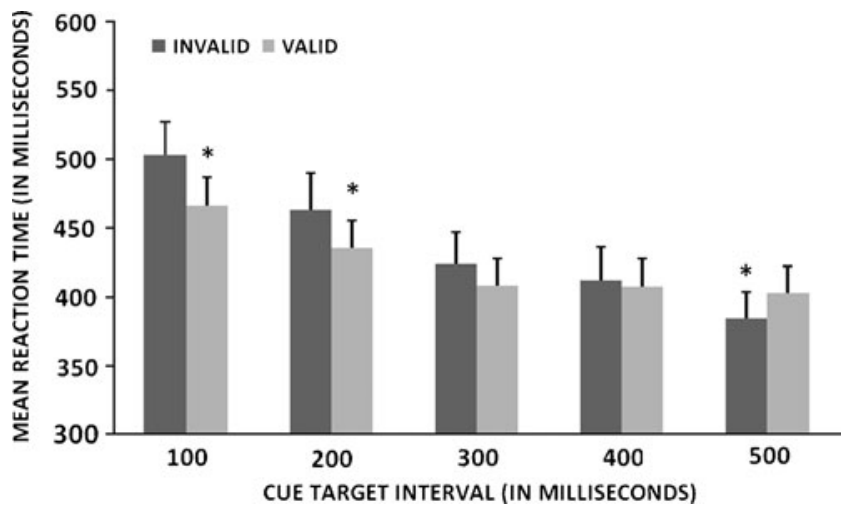

Fig. 2 Mean reaction times as a function of cue-target interval for valid and invalid cued attention in Experiment 1 with a central color cue preceding the peripheral cues on each trial between the onsets of the color cue and the peripheral cues was eliminated. In this way, top-down modulation of WM content was not able to favor one of both colors of the peripheral cues for directing saliency-based attention. This meant that participants needed to orient attention by intentionally comparing left and right peripheral cues with the central cue or by perceptual grouping of equal colors. Note already that we dealt with the issue of perceptual grouping in Experiment 3. If, indeed, saliency-driven orienting processes underlie IOR, we would expecte the reversed cost-benefit pattern to disappear for all CTI conditions.

\section{Method}

Twenty university students (University of Brussels) between 18 and 24 years of age were recruited to participate for course credits. All had normal or corrected-to-normal vision and no color blindness. All participants were naive with respect to the purpose of the study, and none of them had previous experience with cuing tasks. The apparatus, stimuli, procedure, and design were the same as those in Experiment 1, except that the central color cue was now presented simultaneously with the peripheral location cues for $100 \mathrm{~ms}$.

Results

The results of Experiment 2 are presented in Fig. 3. Calculation of mean RTs and the analyses were the same as in the previous experiments.

The effect of CTI was significant, $F(4,76)=87,02, p<$ $.001, \eta_{p}^{2}=.821$. Participants responded more quickly as CTI increased. Over all CTI conditions, a significant cuing effect appeared when the valid and invalid cuing conditions were compared, $F(1,19)=15.61, p<.001, \eta_{p}^{2}=.451$. The interaction between CTI and cue validity appeared significant, $F(4,76)=2.91, p<.05, \eta_{p}^{2}=.133$. 


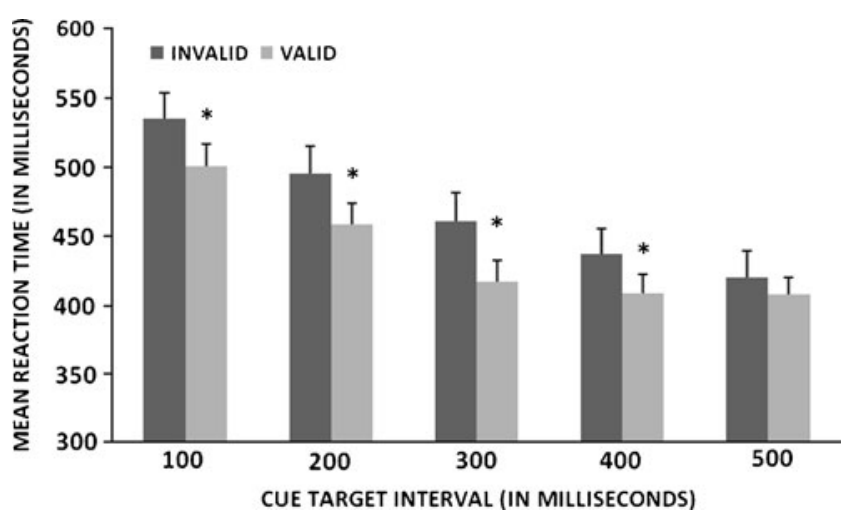

Fig. 3 Mean reaction times as a function of cue-target interval for valid and invalid cued attention in Experiment 2 with simultaneous presentation of central and peripheral cues

Analyses of each CTI condition separately indicated significant cuing effects in all CTI conditions, except for the 500-ms condition, $F(1,19)=1.63$, n.s., $\eta_{p}^{2}=.079$. There was a significant cuing effect of $35 \mathrm{~ms}$ for the 100$\mathrm{ms}$ condition, $F(1,19)=22.76, p<.001, \eta_{p}^{2}=.545,37 \mathrm{~ms}$ for the 200-ms condition, $F(1,19)=11.02, p<.005, \eta_{p}^{2}=$ $.367,43 \mathrm{~ms}$ for the 300 -ms condition, $F(1,19)=13.21, p<$ $.005, \eta_{p}^{2}=.408$, and $27 \mathrm{~ms}$ for the $400-\mathrm{ms}$ condition, $F(1$, 19) $=7.59, p<.05, \eta_{p}^{2}=.285$. Again, the accuracy analysis did not reveal any significant effects. Participants approximated maximum performance level.

Deleting the time between the onset of the color cue and the peripheral cues eliminated IOR in all CTI conditions. A normal cuing pattern was found for all CTI conditions, except the $500-\mathrm{ms}$ condition. This data pattern suggests that the use of saliency-driven visual processes in attentional orienting is essential for IOR to occur. However, it is possible that IOR may emerge with CTIs longer than $500 \mathrm{~ms}$, since the cuing effect is still significant with a 400ms CTI (unlike in Experiment 1). To exclude this possibility, we extended the CTI range for the experimental designs without and with attentional set, in Experiments 4 and 5 , respectively.

Because central and peripheral cues were presented simultaneously, it was possible that participants exploited perceptual grouping of equal colors to automatically direct attention (for a review, see Palmer, 1999). Orienting through perceptual grouping would predict automatically and nonintentionally directing attention by means of bottom-up processing and, accordingly, also the appearance of IOR with the larger CTI conditions. Although the results did not seem to support perceptual grouping, since we did not observe IOR, we nevertheless decided to eliminate the possibility of perceptual grouping in Experiment 3.

\section{Experiment 3: No attentional set (color cue after peripheral cue)}

In Experiment 3, attentional set was prevented by presenting the central color cue after the offset of the peripheral location cue, refuting an explanation of the results of Experiment 2 in terms of perceptual grouping. In this condition, participants were not able to preset attention; neither were they able to use perceptual grouping for directing their attention.

\section{Method}

Eighteen university students (University of Brussels) between 18 and 24 years of age were recruited to participate for course credits. All had normal or correctedto-normal vision and no color blindness. All participants were naive with respect to the purpose of the study, and none of them had previous experience with cuing tasks. The apparatus, stimuli, procedure, and design were the same as those in Experiment 1, except that the central color cue was now presented after the peripheral location cues. The CTI now reflected the time starting from the offset of the central cue until the onset of the target.

\section{Results}

The results of Experiment 3 are presented in Fig. 4. Calculation of mean RTs and the analyses were the same as in the previous experiments.

The overall effect of CTI was significant, $F(4,68)=$ 85.19, $p<.001, \eta_{p}^{2}=.834$. Participants became faster as CTI increased. Over all CTI conditions, there was a significant cuing effect, $F(1,17)=17.92, p<.001, \eta_{p}^{2}=$ .513. The interaction between CTI and cue validity was not significant, $F(4,68)=1.53$, n.s., $\eta_{p}^{2}=.082$.

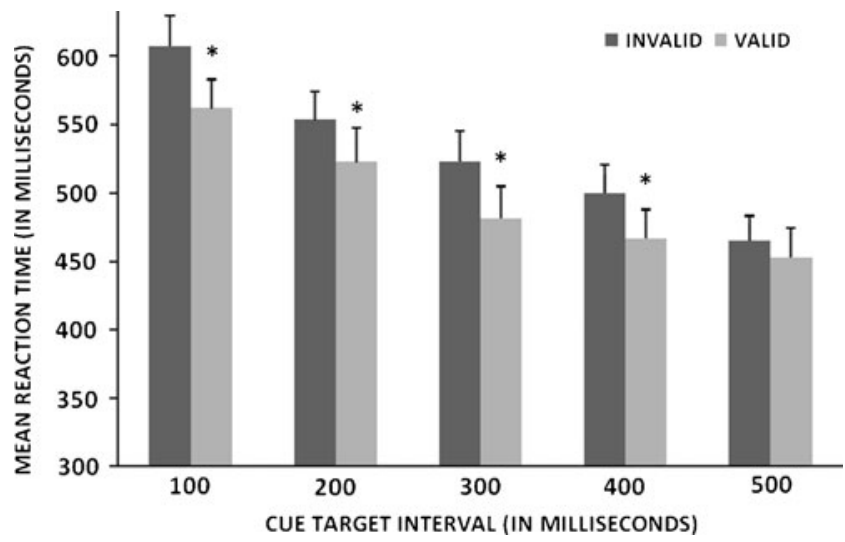

Fig. 4 Mean reaction times as a function of cue-target interval for valid and invalid cued attention in Experiment 3 without attentional presetting (i.e., peripheral cues presentation before central cuing) 
Analyses of each CTI condition separately indicated significant cuing effects in all CTI conditions, except for the 500-ms condition, $F(1,17)=2.84$, n.s., $\eta_{p}^{2}=.143$. That is, a significant cuing effect of $45 \mathrm{~ms}$ was found for the 100-ms condition, $F(1,17)=28.59, p<.001, \eta_{p}^{2}=.627$, an effect of $35 \mathrm{~ms}$ for the 200-ms condition, $F(1,17)=6.00, p<.05, \eta_{p}^{2}=$ .261 , an effect of $46 \mathrm{~ms}$ for the 300-ms condition, $F(1,17)=$ $14.35, p<.001, \eta_{p}^{2}=.458$, and an effect of $38 \mathrm{~ms}$ for the 400 ms condition, $F(1,17)=14.19, p<.005, \eta_{p}^{2}=.455$.

The overall accuracy analysis revealed a marginally significant effect of CTI, $F(4,68)=2.37, p<.10, \eta_{p}^{2}=$ .122. Participants responded less accurately as CTI increased. Over all CTI conditions, a significant cuing effect appeared when the error rates for the valid and invalid cuing conditions were compared, $F(1,17)=5.14, p<.05, \eta_{p}^{2}=$ .232. That is, participants were less accurate in the invalid cuing condition than in the valid cuing condition. The interaction between CTI and cue validity was not significant, $F(4,68)=1.25$, n.s., $\eta_{p}^{2}=.068$.

Accuracy analyses of each CTI condition separately indicated significant cuing effects in all CTI conditions, except for the 300 -ms condition, $F(1,17)=2.24$, n.s., $\eta_{p}^{2}=.117$, and the 500-ms condition, $F(1,17)=0.01$, n.s., $\eta_{p}^{2}=.001$. In the 100-ms condition, participants were less accurate in the invalid cuing condition ( $97 \%$ correct) than in the valid cuing condition $\left(100 \%\right.$ correct), $F(1,17)=10.90, p<.005, \eta_{p}^{2}=$ .391. In the 200-ms condition, invalid cuing resulted in a mean accuracy of $96 \%$, which significantly differed from the valid cuing accuracy of $99 \%, F(1,17)=5.30, p<.05, \eta_{p}^{2}=$ .238. Finally, in the 400-ms condition, the invalid cuing condition with an accuracy of $96 \%$ significantly differed from the valid cuing condition with an accuracy of $99 \%, F(1,17)=$ 4.78, $p<.005, \eta_{p}^{2}=.220$.

The need for remembering the peripheral color cues in short-term memory complicated the task, so that it was possible to observe a cuing effect in the accuracy data, as well as in the RT data. The pattern of the accuracy data supported the findings in the RT data to some extent. If a cuing effect was found, performance in the valid condition was more accurate than in the invalid cuing condition. In the 500-ms condition, the cuing effect disappeared in both RT and error analyses.

\section{Extended CTI range}

We replicated Experiments 1 and 2 with an extended CTI range from 100 to $900 \mathrm{~ms}$. That is, in Experiment 4, we wanted to address the possibility that, in Experiment 2 (without attentional set), IOR would have appeared with longer CTIs. If IOR is related to saliency-based orienting processes, no IOR will be expected without the possibility of attentional set, even when CTI range is extended. In Experiment 5, we replicated Experiment 1 (with attentional set), to control for the effect of an extended CTI range on cuing effects. We expected a clear IOR effect also for the longer CTIs, because endogenously generated IOR seemed to be delayed to longer CTIs, as compared with exogenous cuing tasks (Experiment 1).

\section{Experiment 4 (no attentional set)}

Method

Twenty-one university students (University of Brussels) between 18 and 24 years of age were recruited to participate for course credits. All had normal or correctedto-normal vision and no color blindness. All participants were naive with respect to the purpose of the study, and none of them had previous experience with cuing tasks. The apparatus, stimuli, procedure, and design were the same as those in Experiment 2, except that the CTI conditions were now $100,300,500,700$, or $900 \mathrm{~ms}$.

\section{Results}

The results of Experiment 4 are presented in Fig. 5. Calculation of mean RTs and the analyses were the same as in the previous experiments.

The overall effect of CTI was significant, $F(4,80)=$ 77.48, $p<.001, \eta_{p}^{2}=.795$. Participants responded more quickly as CTI increased. There was also a significant main effect of cuing, $F(1,20)=12.44, p<.005, \eta_{p}^{2}=.383$, and an interaction between CTI and cue validity, $F(4,80)=$ $10.78, p<.001, \eta_{p}^{2}=.350$.

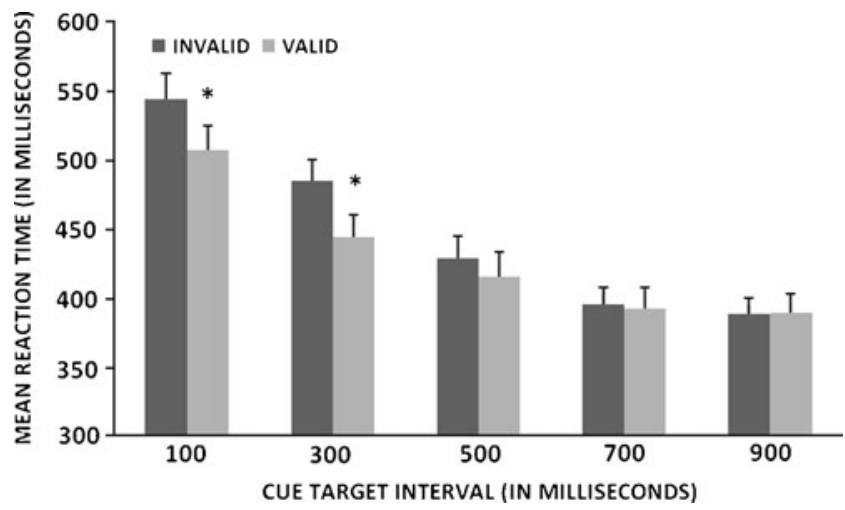

Fig. 5 Mean reaction times as a function of cue-target interval for valid and invalid cued attention in Experiment 4 with simultaneous presentation of central and peripheral cues and with extended CTI range 
Analyses of each CTI condition separately indicated significant cuing effects in the $100-\mathrm{ms}$ condition $(37 \mathrm{~ms})$, $F(1,20)=45.32, p<.001, \eta_{p}^{2}=.694$, and the $300-\mathrm{ms}$ condition (41 ms), $F(1,20)=45.85, p<.001, \eta_{p}^{2}=.696$. No cuing effect was found in the 500-ms condition, $F(1,20)=2.15$, n.s., $\eta_{p}^{2}=.097$, the $700-\mathrm{ms}$ condition, $F(1,20)=0.09$, n.s., $\eta_{p}^{2}=.004$, and the $900-\mathrm{ms}$ CTI condition, $F(1,20)=0.02$, n.s., $\eta_{p}^{2}=.001$.

RT patterns in the present experiment without attentional set confirmed the RT pattern in Experiment 2. With CTIs of $500 \mathrm{~ms}$ and longer, there was no cuing effect and no IOR. RTs for both valid, $t(20)=0.54$, n.s., and invalid, $t(20)=$ 1.5 , n.s., cuing did not differ when the CTI was enlarged to 700 and $900 \mathrm{~ms}$. Accordingly, no IOR would be expected through (further) manipulating the CTI in the present endogenous cuing experiment without attentional set.

As in Experiments 1 and 2, participants approximated the maximum performance level of accuracy in all conditions. There were no significant differences in the accuracy data.

\section{Experiment 5: Attentional set}

\section{Method}

Twenty-one university students (University of Brussels) between 18 and 24 years of age were recruited to participate for course credits. All had normal or correctedto-normal vision and no color blindness. All participants were naive with respect to the purpose of the study, and none of them had previous experience with cuing tasks. The apparatus, stimuli, procedure, and design were the same as those in Experiment 1, except that the CTI conditions were now100, 300, 500, 700, and $900 \mathrm{~ms}$.

\section{Results}

The results of Experiment 5 are presented in Fig. 6 . Calculation of mean RTs and the analyses were the same as in the previous experiments.

The overall effect of CTI was significant, $F(4,80)=$ $66.81, p<.001, \eta_{p}^{2}=.770$. Participants responded more quickly as CTI increased. Over all CTI conditions, no cuing effect was found when the valid and invalid trials were compared, $F(1,20)=1.29$, n.s., $\eta_{p}^{2}=.061$. The interaction between CTI and cue validity was clearly significant, $F(4,80)=24.74, p<.001, \eta_{p}^{2}=.553$.

Analyses of each CTI condition separately indicated significant cuing effects in the 100 -ms condition $(31 \mathrm{~ms})$, $F(1,20)=39.47, p<.001, \eta_{p}^{2}=.664$, and the $300-\mathrm{ms}$

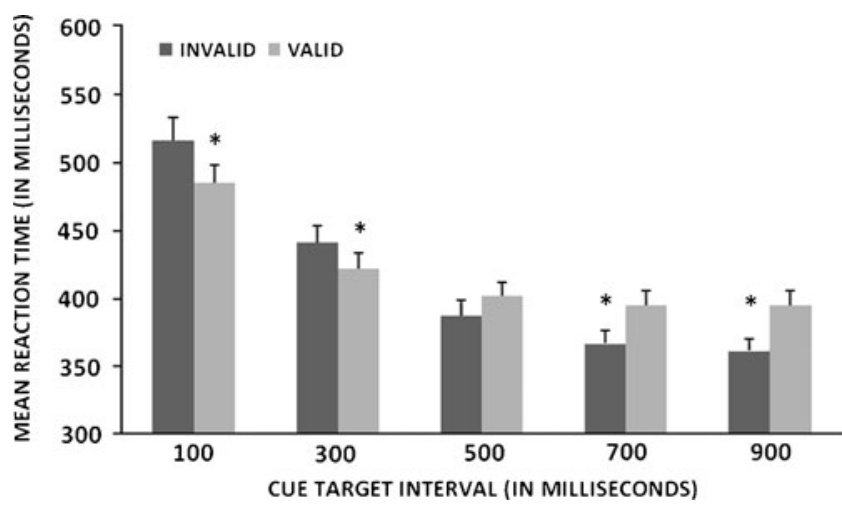

Fig. 6 Mean reaction times as a function of cue-target interval for valid and invalid cued attention in Experiment 5 with a central color cue preceding the peripheral cues on each trial and with extended CTI range

condition (19 ms), $F(1,20)=12.57, p<.005, \eta_{p}^{2}=.386$. In the 500-ms CTI condition, no effect of validity was observed, $F(1,20)=2.69$, n.s., $\eta_{p}^{2}=.119$. A reversed cuing pattern was found for a CTI of $700 \mathrm{~ms}(-28 \mathrm{~ms}), F(1,20)=$ 15.01, $p<.001, \eta_{p}^{2}=.429$, and for $900 \mathrm{~ms}(-33 \mathrm{~ms})$, $F(1,20)=21.82, p<.001, \eta_{p}^{2}=.522$. In the latter conditions, valid cuing resulted in longer RTs, as compared with invalid cuing.

Manipulation of the CTI beyond the 500-ms limit of Experiment 1 confirmed the finding of an IOR pattern with endogenous cuing through attentional set for the larger CTIs. However, in contrast with Experiment 1, the transition from a normal cuing pattern to a reversed costbenefit pattern seemed to be delayed for a CTI of $700 \mathrm{~ms}$.

The accuracy analysis again did not reveal any significant effects. In all conditions, participants approximated the maximum performance level.

\section{Experiment 6: Attentional set by means of color words}

With a final experiment, we wanted to address the possibility that IOR in Experiments 1 and 5 was due to exogenous attentional processes caused by perceptual priming or grouping, instead of orienting through endogenously biasing the bottomup saliency processes. First, one could argue that the color of the peripheral cue was perceptually primed by the color of the preceding central cue, causing an exogenous attentional shift. Second, with fast succession of central and peripheral color cues, it may also be that equal colors are automatically and unintentionally grouped (perceptual grouping; for a review, see Palmer, 1999). Accordingly, the endogenous bias of saliency processing with our split-cuing paradigm would no longer account for the appearance of IOR. To ensure that the appearance of IOR in Experiments 1 and 5 was not caused by low-level priming or perceptual grouping effects, we used 
a neutral-colored (i.e., gray) color word as the central instructional cue in Experiment 6. The use of a written color word cue eliminated low-level automatic priming, since the cue words needed to be interpreted. Next, perceptual grouping effects were impossible, since central and peripheral cues were perceptually dissimilar. Finally, changing the color word cue on each trial eliminated the possibility of intertrial priming. Using the association between our lexicon and the perceptual color in the split-cue paradigm may also better resemble reality. That is, when we are intentionally searching for a particular object among others - for example, a particular shirt in the closet—we may first (semantically) think over the feature that distinguishes the object from other objects in order to bias it (e.g., I know the particular shirt is red). Note that although the use of color words as a central cue eliminates perceptual priming, it may now cause cross-modal semantic color priming (Gibson, 1966). However, there is a difference between both modes of priming that is of relevance in the present study. Similar to the use of endogenous arrow cues (or spatial words "left" and "right") with semantic primes, participants need to apply top-down knowledge to the prime or cue. That is, participants need decode (i.e., interpret) the word or symbol to be able to use it. The processing of exogenous cues and perceptual primes, on the other hand, does not rely on high-level decoding.

We would predict that if IOR in Experiments 1 and 5 was indeed due to low-level priming, excluding possible priming and grouping requisites should cause IOR to disappear. Yet, if IOR was due to top-down presetting of bottom-up saliency, we would expect the IOR effect to appear with the larger CTI conditions.

\section{Method}

Participants, apparatus, stimuli, and procedure Thirtythree university students (University of Brussels) between 18 and 24 years were recruited to participate for course credits. All had normal or corrected-to-normal vision and no color blindness. All participants were naive with respect to the purpose of the study, and none of them had previous experience with cuing tasks. The apparatus, stimuli, procedure, and design were the same as those in Experiment 5, except that the central color cue was replaced by a 1,000 -msappearing color word cue. The color word varied from trial to trial and semantically referred to which of the two peripheral color cues the participant needed to orient his or her attention. To also exclude the possibility of intertrial distractor priming, distractor colors (white, blue, yellow, and cyan) were also varied over two successive trials.

Design After the instructions, a practice block of 40 trials was presented. When practice accuracy was above $85 \%$, 240 experimental trials were presented. After each series of
60 trials, a 10-s break was inserted. Cue validity and CTI were manipulated randomly within subjects. Targets were randomly and equally distributed over both locations for all conditions. Critical dependent variables were RT and accuracy.

\section{Results}

The results of Experiment 6 are presented in Fig. 7 . Calculation of mean RTs and the analyses were analogous to those in the previous experiments.

The overall effect of CTI was significant, $F\left(\begin{array}{ll}4,1 & 28\end{array}\right)=$ 231,93, $p<.001, \eta_{p}^{2}=.879$. Participants responded more quickly as CTI increased. No main effect of cuing was observed over all CTI conditions, $F(1,32)=0.22$, n.s., $\eta_{p}^{2}=$ .007 . The interaction between CTI and cue validity was significant, $F(4,128)=17.74, p<.001, \eta_{p}^{2}=.357$.

Analyses of each CTI condition separately indicated a significant cuing effect in the $100-\mathrm{ms}$ condition $(28 \mathrm{~ms}), F(1$, $32)=33.30, p<.001, \eta_{p}^{2}=.510$. In the $300-m s$ condition, a marginally significant cuing effect was observed (14 ms), $F(1,32)=3.61, p<.07, \eta_{p}^{2}=.101$. In the 500-ms CTI condition, no effect of validity was observed, $F(1,32)=$ 2.24 , n.s., $\eta_{p}^{2}=.065$. A significant reversed cuing pattern was observed in the $700-\mathrm{ms}$ condition $(-18 \mathrm{~ms}), F(1,32)=5.73$, $p<.05, \eta_{p}^{2}=.152$, and in the 900-ms CTI condition, (-23 ms), $F(1,32)=15.54, p<.001, \eta_{p}^{2}=.327$ - that is, valid cuing resulting in longer RTs, as compared with invalid cuing.

The RT results of this control experiment using color words indicated that the observation of the IOR phenomenon with our endogenous split-cue design (Experiments 1 and 5) was not due to low-level priming. That is, eliminating the prerequisites for low-level priming did not

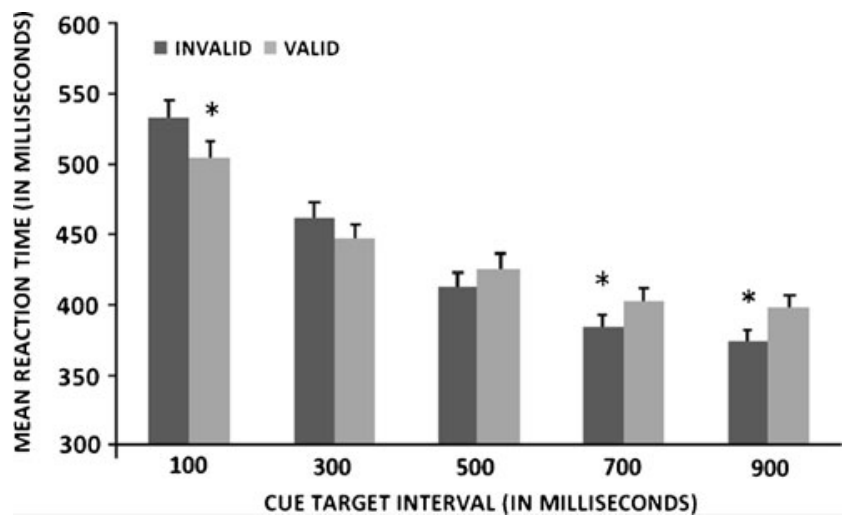

Fig. 7 Mean reaction times as a function of cue-target interval for valid and invalid cued attention in Experiment 6 with an instructional color word cue 
result in the disappearance of IOR. Accordingly, the suggestion that IOR is related to low-level saliency processes that can be endogenously modulated (Experiments 1 and 5) can be maintained.

As in the previous experiment, the accuracy analysis did not reveal any significant effects.

\section{General discussion}

In the present study, we set out from the assumption that the absence of IOR in most endogenous cuing tasks, as compared with exogenous cuing, is caused by the difference in processing level where the cuing effects are assumed to be active. Whereas exogenous cues act upon stimulus saliency, which is associated with low-level stimulus processing, endogenous cues are mostly associated with higher-level processing, involving the task set in WM. If IOR is a process linked to mechanisms of low-level saliency processing, it can occur only when cues interact with low-level processes. To investigate this hypothesis, we designed a series of experiments with a split-cuing technique in order to create endogenous cuing conditions with and without low-level saliency processing. The results clearly showed IOR in conditions that addressed low-level saliency processes to orient attention. This supported the claim that attentional control by saliency is a prerequisite for IOR.

In general, the IOR phenomenon is found with exogenously cued attention and only with endogenous cuing when eye movements are programmed. Because covert exogenous orienting and oculomotor programming share the use of bottom-up saliency information (Clark, 1999; Findlay, 2009; Itti \& Koch, 2000, 2001), IOR may well be linked to these low-level saliency processes. In exogenous attentional selection, for example, orienting is controlled stimulus-driven, through the bottom-up processing of the external stimuli. With endogenous attentional selection, orienting is controlled intentionally, through top-down guidance. Under traditional covert endogenous cuing conditions (not accompanied by eye movements), bottomup low-level saliency processes are not involved in orienting attention. Accordingly, it is likely that no welldefined location is favored in the early visual map. That is, endogenously cued attention may not be immediately directed to the cued location but, rather, searches for the target object while orienting in the cued direction. Consequently, the possible target (or "cued") location itself often may not be activated in the visual map before target onset, but rather the direction of target appearance is cued. Thus, attention will be guided endogenously in the cued direction, while suppressing or ignoring bottom-up attentional signals from the low-level saliency map.
The split-cuing technique was designed to make endogenous attention act upon low-level saliency processes. That is, a central color cue instructed participants as to which peripheral location they needed to orient-namely, the location containing the peripheral cue with the same color as the central instruction cue. By presenting the central cue before the appearance of the peripheral cue, participants could store the central cue feature in WM and prepare for orienting to the instructed peripheral location through a topdown bias of WM activation on the saliency maps (e.g., Olivers et al., 2006). We assumed that orienting could be guided by this selective prioritization in saliency processing (attentional set) only when there was sufficient time between the onset of the central and the peripheral cues. When low-level saliency processing is indeed essential for IOR to occur, we would expect a reversed cost-benefit pattern for the larger CTI conditions in experiments with attentional set (Experiments 1, 5, and 6) and the absence of IOR in experiments without attentional set (Experiments 2, 3 , and 4).

In Experiment 1, which was designed to endogenously activate saliency processes (attentional set), a facilitatory cuing effect was found for the short CTI conditions and an IOR effect for the longer CTI conditions. This supported our suggestion that saliency processing may, indeed, be a requisite for the appearance of IOR. However, IOR onset was delayed in the 500-ms CTI condition, while the reversal already appeared after about the 250-ms CTI in most exogenous cuing experiments (e.g., Posner \& Cohen, 1984). This discrepancy may have been caused by the difference in processing an endogenous split cue versus an exogenous cue. That is, with exogenous cue instructions, participants were told to ignore the cue, which automatically captured attention, and to respond only to the target onset as quickly as possible. Focusing and processing the exogenous cue were reduced as much as possible, since the cue and its features wereconsidered "redundant" for the task. In contrast, with endogenous split cuing, participants were instructed to voluntarily orient attention to the location of the peripheral cue that shared its color with the central cue (Experiments 1-5) or written cue (Experiment 6). In general, the importance of the cue was stressed. Accordingly, participants intentionally focused on the peripheral cue sharing the particular "task-related" feature. Intentionally processing the peripheral cue may have caused an increase in the time to disengage attention from the endogenous peripheral split cue whose features were "task-relevant," as compared with an exogenous "task redundant" cue. The longer disengagement time with taskrelevant cues could account for the delay of IOR.

Previous studies did show a delay of endogenous cuing, as compared with exogenous cuing, but for the facilitatory effect (e.g., Jonides \& Irwin, 1981; Müller \& Findlay, 1988; 
Müller \& Rabbitt, 1989; Rafal \& Henik, 1994). In the present study, we were not able to observe a delay in facilitatory effects with the endogenous split-cue task. A possible explanation concerns the location at which the attentional disengagement operates. With a traditional endogenous cue (e.g., a central arrow), slower attentional disengagement would delay orienting from the center of the screen to the cued location. Accordingly, the facilitatory cuing effect is delayed. In contrast, with an endogenous split cue, slower attentional disengagement from the peripheral cue does not affect facilitatory effects, since the peripheral cue location corresponds with the cued location. Accordingly, the spatial location of attentional disengagement (i.e., central vs. peripheral) could explain why, with the split-cue design, only IOR was delayed, while the facilitatory cuing effect remained unchanged. Finally, it may also be important to note that the gray target stimulus used in the present study was perceptually less conspicuous, as compared with the preceding colored cues. Castel, Pratt, Chasteen, and Scialfa (2005) found that perceptually degraded targets increase task difficulty and, in this way, affect the time course of IOR.

Experiment 2 was conducted with simultaneous presentation of central color and peripheral location cues to prevent the formation of an attentional set prior to the presentation of the peripheral cues. In these circumstances, participants had no time to store the relevant color in WM, and consequently, no top-down presetting of the low-level saliency maps was possible. Participants' orientation was expected to be endogenously guided, without modulation of bottom-up saliency processing. The simultaneous presentation of both cues again resulted in early facilitatory effects, indicating that participants did make use of the cues. However, the transition from facilitation to IOR for the longer CTI conditions no longer appeared. This clearly supported our claim that IOR occurs only when low-level saliency processes are claimed in the orienting process. An alternative explanation for the results of Experiment 2 was that perceptual grouping, or the predominance of the central cue color in the visual field (due to simultaneous presentation of central and peripheral cues), would guide attention automatically to the cued location.

Therefore, in Experiment 3, we eliminated this possibility by presenting the peripheral cue before the central instructional cue. Results again supported the suggestion that without the opportunity to modulate low-level bottomup saliency processes, no IOR effect is possible in attentional orienting. As in Experiment 2, the cuing effect was still significant with a CTI of $400 \mathrm{~ms}$, and no reversed cuing patterns were observed in RT. In contrast with the previous experiments, we did, however, observe a positive cuing effect in the accuracy data, which may have been caused by an increase in task demands. Surprisingly, we did not find a difference in mean accuracy between Experiments 2 and 3 . Yet there was a clear difference in mean RTs. That is, mean RTs in Experiment 2 were significantly shorter, as compared with Experiment $3(66 \mathrm{~ms}), F(1,36)=$ $6,23, p<.02$. Taking into account that participants could lose course credits when the error rate was too high, it is not surprising that in a more difficult task, a speed-accuracy trade-off affected the effect validity in the accuracy data.

In Experiments 2 and 3, the possibility remained that IOR would emerge with CTIs longer than $500 \mathrm{~ms}$. Therefore, in Experiment 4, we replicated Experiment 2 (without attentional set; simultaneous split cue) with an extended range of CTIs from 100 to $900 \mathrm{~ms}$. Also, with CTIs beyond $500 \mathrm{~ms}$ (i.e., 700 and $900 \mathrm{~ms}$ ), no IOR appeared in the RT pattern. To allow a comparison of these results with an analogous endogenous orienting experiment that encouraged the use of saliency information (with attentional set), also the range of the CTIs in Experiment 1 was extended in Experiment 5. Here again, IOR was observed with the larger CTIs, which confirmed the suggestion regarding the need for saliency processing in attentional inhibitory tagging. It is also noteworthy that, with an enlarged CTI range, the moment of transition from facilitatory cuing effects to inhibitory effects seemed to be influenced. The inhibitory effect appeared only with a CTI of 700 and $900 \mathrm{~ms}$, whereas in the experiments with a shorter CTI range (Experiment 1), the reversed cuing effect was already significant with a 500-ms CTI. The shift to a later transition time can be interpreted as support for the findings of Taylor (2007), who demonstrated the modulation of IOR by cognitive top-down expectancies (see also Henderickx, Maetens, \& Soetens, 2011; Los, 2004). The extended CTI range probably created expectancies for a later possible target onset, as compared with experiments with a shorter CTI range, and this in turn may have induced the postponement of the inhibitory effect.

Finally, in Experiment 6 we used a gray color word as the central instructional cue to address the possibility that the observed IOR effect in Experiments 1 and 5 was due to exogenous orienting resulting from perceptual priming or grouping, instead of endogenous orienting. Although priming and grouping requisites were eliminated, again an IOR effect was found for the longer CTI conditions. In sum, the results in the present study suggest that the cuing effect in endogenous orienting is subject to whether or not low-level saliency processes have been used to guide attentional orienting. When endogenous attentional orienting was guided by an attentional set that favored a particular feature in low-level saliency processing (Experiments 1,5 , and 6), a reversal of the cost-benefit pattern was observed when CTI was prolonged. That is, facilitative processing at the cued location with short CTIs due to attentional focusing was followed by an inhibitory process 
restraining attentional orientation to the previously attended location with longer CTIs.

Consistent with an attentional account in the explanation of IOR (Klein, 1988; Lupiáñez et al., 2004; Posner \& Cohen, 1984), the present suggestions support the possibility that IOR reflects inhibited sensory/perceptual processing at the cued location. That is, IOR seems to reflect a bias of attention toward novel locations and against returning to previously attended objects and locations. Nevertheless, saliency processing is also assumed to be closely related to the oculomotor system regarding the planning of voluntary saccades (Clark, 1999) and the planning of involuntary saccades to exogenously salient targets (Findlay, 2009). Therefore, the appearance of IOR with cuing conditions in which saliency processing is used to orient attention supports the idea that both the oculomotor system (motor account) and the attentional system (attentional account) are triggered in conditions under which IOR occurs (Dorris et al., 2002; Kingstone \& Pratt, 1999; Mayer et al., 2004; Sapir et al., 1999).

Posner et al. (1985) and Rafal et al. (1989) suggested that saccade programming is a necessary condition for IOR to occur. Because saliency processing is assumed to be indispensable for saccades (Clark, 1999; Findlay, 2009), the present results, suggesting a close relationship between saliency processing and the emergence of IOR, seem to support this claim. However, with the present findings, a somewhat more prudent conclusion seems preferable (see also Chica, Klein, Rafal, \& Hopfinger, 2010). Because we have no measures for saccade programming or execution in the present experimental design, we cannot fully disentangle the issue of whether activation of the oculomotor system itself is a necessary condition for the appearance of IOR. Nevertheless, despite the fact that eye movements were not prohibited in the control experiments, no IOR was observed. For now, an explanation in terms of the importance of saliency processing for both (most) oculomotor programming and IOR seems to be most straightforward. Note that it is likely that particular eye movements can be triggered without activation of the saliency maps. That is, when no target location or target feature is inherently salient or has become salient through top-down modulation. For example, when voluntary eye movements are planned in an environment with identical objects or no objects at all, the use of saliency information is ruled out (e. g., Chica et al., 2010). This could explain the absence of IOR in many endogenous cuing studies, even though eye movements were possible (e.g., Briand, 1998; Briand \& Klein, 1987; Henderickx, Maetens, \& Soetens, 2010) or saccades were intentionally prepared (Chica et al., 2010).

The explanation in terms of saliency processing harmonizes with the suggestion of Dorris et al. (2002) and Godijn and Theeuwes (2004) that the inhibition causing IOR acts not upon the saccade map in which oculomotor programming occurs (most likely the SC) but, rather, within a system that provides input to the saccade map (see also Theeuwes et al., 2006). In accordance with the suggestion that IOR is caused by saliency processing, a number of studies have suggested that the lateral intraparietal area (the preoculomotor attention map in Godijn \& Theeuwes, 2004) is involved in the generation of attentional orienting (e.g., Gottlieb \& Goldberg, 1999). It is proposed that activity in this area acts as a saliency representation (Bisley \& Goldberg, 2003; Gottlieb, Kusunoki, \& Goldberg, 1998) and can be modulated by local and global top-down mechanisms (Balan \& Gottlieb, 2006). In accordance with the theory of Godijn and Theeuwes (2004) and neurological findings/theories (e.g., Balan \& Gottlieb, 2006; Dorris et al., 2002; Shipp, 2004; Vivas, Humphreys, \& Fuentes, $2003,2006,2008$ ), we suggest that IOR is likely caused in the lateral intraparietal area of the posterior parietal cortex, which acts as a retinotopic visual saliency map and can be modulated through working memory.

In closing, it may also be interesting to note that it has once more been demonstrated that the terms endogenous and exogenous processes, on the one hand, and top-down and bottom-up processes, respectively, on the other hand, cannot be treated as synonyms. That is, endogenous orienting, which has usually been associated only with top-down processing, may also act upon bottom-up processes. In the present study, a top-down bias of bottom-up saliency processing resulted in the appearance of IOR with endogenous orienting. Accordingly, the use of low-level saliency processes in attentional orienting is suggested to be a prerequisite for the appearance of IOR.

\section{References}

Balan, P. F., \& Gottlieb, J. (2006). Integration of exogenous input into a dynamic salience map revealed by perturbing attention. Journal of Neuroscience, 26, 9239-9249.

Bisley, J. W., \& Goldberg, M. E. (2003). Neuronal activity in the lateral intraparietal area and spatial attention. Science, 299, 81-86.

Briand, K. A. (1998). Feature integration and spatial attention: More evidence of a dissociation between endogenous and exogenous orienting. Journal of Experimental Psychology: Human Perception and Performance, 24, 1243-1256.

Briand, K. A., \& Klein, R. M. (1987). Is Posner's "beam" the same as Treisman's "glue"? On the relation between visual orienting and feature integration theory. Journal of Experimental Psychology: Human Perception and Performance, 13, 228-241.

Castel, A. D., Pratt, J., Chasteen, A. L., \& Scialfa, C. T. (2005). Examining task difficulty and the time course of inhibition of return: Detecting perceptually degraded targets. Canadian Journal of Experimental Psychology, 59, 90-98.

Cave, K. R., \& Wolfe, J. M. (1990). Modeling the role of parallel processing in visual search. Cognitive Psychology, 22, 225-271.

Chica, A. B., Klein, R. M., Rafal, R. D., \& Hopfinger, J. B. (2010). Endogenous saccade preparation does not produce inhibition of 
return: Failure to replicate Rafal, Calabresi, Brennan, \& Sciolto (1989). Journal of Experimental Psychology: Human Perception and Performance, 36, 1193-1206.

Clark, J. J. (1999). Spatial attention and latencies of saccadic eye movements. Vision Research, 39, 585-602.

Danziger, S., Ward, R., Owen, V., \& Rafal, R. (2002). The effects of unilateral pulvinar damage in humans on reflexive orienting and filtering of irrelevant information. Behavioral Neurology, 13, 95104.

Desimone, R., \& Duncan, J. (1995). Neural mechanisms of selective visual attention. Annual Review of Neuroscience, 18, 193-222.

Dorris, M. C., Klein, R. M., Everling, S., \& Munoz, D. P. (2002). Contribution of the primate superior colliculus to inhibition of return. Journal of Cognitive Neuroscience, 14, 1256-1263.

Findlay, J. M. (2009). Saccadic eye movement programming: Sensory and attentional factors. Psychological Research, 73, 127-135.

Folk, C. L., \& Remington, R. W. (1998). Selectivity in attentional capture by featural singletons: Evidence for two forms of attentional capture. Journal of Experimental Psychology: Human Perception and Performance, 24, 847-858.

Folk, C. L., \& Remington, R. W. (1999). Can new objects override attentional control settings? Perception \& Psychophysics, 61, 727-739.

Folk, C. L., \& Remington, R. W. (2006). Top-down modulation of preattentive processing: Testing the recovery account of contingent capture. Visual Cognition, 14, 445-465.

Folk, C. L., Remington, R. W., \& Johnston, J. C. (1992). Involuntary covert orienting is contingent on attentional control settings. Journal of Experimental Psychology: Human Perception and Performance, 18, 1030-1044.

Folk, C. L., Remington, R. W., \& Wright, J. H. (1994). The structure of attentional control: Contingent attentional capture by apparent motion, abrupt onset, and colour. Journal of Experimental Psychology: Human Perception and Performance, 20, 317-329.

Gibson, J. J. (1966). The senses considered as perceptual systems. Boston: Houghton Mifflin.

Godijn, R., \& Theeuwes, J. (2004). The relationship between inhibition of return and saccade trajectory deviations. Journal of Experimental Psychology: Human Perception and Performance, 30, 538-554.

Gottlieb, J., \& Goldberg, M. E. (1999). Activity of neurons in the lateral intraparietal area of the monkey during an antisaccade task. Nature Neuroscience, 2, 906-912.

Gottlieb, J., Kusunoki, M., \& Goldberg, M. E. (1998). The representation of visual salience in monkey parietal cortex. Nature, 391, 481-484.

Henderickx, D., Maetens, K., Geerinck, T., \& Soetens, E. (2009). Modeling the interactions of bottom-up and top-down guidance in visual attention. In L. Paletta \& J. K. Tsotsos (Eds.), Attention in cognitive systems (pp. 197-211). Berlin: Springer.

Henderickx, D., Maetens, K., \& Soetens, E. (2010). Feature integration and spatial attention: Common processes for endogenous and exogenous orienting. Psychological Research, 74, 239-254.

Henderickx, D., Maetens, K., \& Soetens, E. (2011). Strategic modulation of inhibition of return: The effect of temporal target onset predictability in detection and discrimination tasks. Manuscript submitted for publication.

Itti, L., \& Koch, C. (2000). Saliency-based search mechanism for overt and covert shifts of visual attention. Visual Research, 40, $1489-1506$.

Itti, L., \& Koch, C. (2001). Computational modeling of visual attention. Nature Reviews Neuroscience, 2, 194-203.

Jonides, J. (1981). Voluntary vs. automatic control over the mind's eye's movement. In J. B. Long \& A. D. Baddeley (Eds.),
Attention and performance IX (pp. 187-203). Hillsdale, NJ: Erlbaum.

Jonides, J., \& Irwin, D. E. (1981). Capturing attention. Cognition, 10, $145-150$.

Jonides, J., \& Yantis, S. (1988). Uniqueness of abrupt stimulus onset in capturing attention. Perception \& Psychophysics, 43, 346-354.

Kingstone, A., \& Pratt, J. (1999). Inhibition of return is composed of attentional and oculomotor processes. Perception \& Psychophysics, 61, 1046-1054.

Klein, R. M. (1988). Inhibitory tagging system facilitates visual search. Nature, 334, 430-431.

Klein, R. M., \& MacInnes, W. J. (1999). Inhibition of return is a foraging facilitator in visual search. Psychological Science, 10, 346-352.

Koch, C., \& Ullman, S. (1985). Shifts in selective visual attention: Towards the underlying neural circuitry. Human Neurobiology, 4, 219-227.

Los, S. A. (2004). Inhibition of return and nonspecific preparation: Separable inhibitory control mechanisms in space and time. Perception \& Psychophysics, 66, 119-130.

Lupiáñez, J., Decaix, C., Siéroff, E., Chokron, S., Milliken, B., \& Bartolomeo, P. (2004). Independent effects of endogenous and exogenous spatial cueing: Inhibition of return at endogenous attended target locations. Experimental Brain Research, 159, $447-457$

Mayer, A. R., Seidenberg, M., Dorflinger, J. M., \& Rao, S. M. (2004). An event-related fMRI study of exogenous orienting: Supporting evidence for the cortical basis of inhibition of return? Journal of Cognitive Neuroscience, 16, 1262-1271.

Müller, H. J., \& Findlay, J. M. (1988). The effect of visual attention on peripheral discrimination thresholds in single and multiple element displays. Acta Psychologica, 69, 129-155.

Müller, H. J., \& Rabbitt, P. M. A. (1989). Reflexive and voluntary orienting of visual attention: Time course of activation and resistance to interruption. Journal of Experimental Psychology: Human Perception and Performance, 15, 315-330.

Müller, H. J., \& von Mühlenen, A. (2000). Probing distractor inhibition in visual search: Inhibition of return. Journal of Experimental Psychology: Human Perception and Performance, 26, 1591-1605.

Navalpakkam, V., \& Itti, L. (2005). Modeling the influence of task on attention. Vision Research, 45, 205-231.

Olivers, C. N. L., Meijer, F., \& Theeuwes, J. (2006). Feature-based memory-driven capture: Visual working memory content affects visual attention. Journal of Experimental Psychology: Human Perception and Performance, 32, 1243-1265.

Palmer, S. (1999). Vision science: Photons to phenomenology. Cambridge, MA: MIT Press.

Posner, M. I. (1980). Orienting of attention. Quarterly Journal of Experimental Psychology, 32, 3-25.

Posner, M. I., \& Cohen, Y. (1984). Components of visual orienting. In H. Bouma \& D. G. Bouwhuis (Eds.), Attention and performance X: Control of language processes (pp. 55-66). Hillside, NJ: Erlbaum.

Posner, M. I., Rafal, R. D., Choate, L., \& Vaughan, J. (1985). Inhibition of return: Neural basis and function. Cognitive Neuropsychology, 2, 211-228.

Rafal, R. D., Calabresi, P. A., Brennan, C. W., \& Sciolto, T. K. (1989). Saccade preparation inhibits reorienting to recently attended locations. Journal of Experimental Psychology: Human Perception and Performance, 15, 673-685.

Rafal, R. D., \& Henik, A. (1994). The neurology of inhibition: integrating controlled and automatic processes. In D. E. Dagenbach \& T. H. Carr (Eds.), Inhibitory processes in attention, memory, and language (pp. 1-51). San Diego, CA: Academic.

Remington, R. W., Johnston, J. C., \& Yantis, S. (1992). Involuntary attentional capture by abrupt onsets. Perception \& Psychophysics, 51, 279-290. 
Sala, J. B., \& Courtney, S. M. (2009). Flexible working memory representation of the relationship between an object and its location as revealed by interactions with attention. Attention, Perception, \& Psychophysics, 71, 1525-1533.

Sapir, A., Hayes, A., Henik, A., Danziger, S., \& Rafal, R. (2004). Parietal lobe lesions disrupt saccadic remapping of inhibitory location tagging. Journal of Cognitive Neuroscience, 16, 1-7.

Sapir, A., Soroker, N., Berger, A., \& Henik, A. (1999). Inhibition of return in spatial attention: Direct evidence for collicular generation. Nature Neuroscience, 2, 1053-1054.

Schneider, W., Eschman, A., \& Zuccolotto, A. (2002). Prime user's guide. Pittsburgh, PA: Psychology Software Tools.

Shipp, S. (2004). The brain circuitry of attention. Trends in Cognitive Sciences, 8, 223-230.

Soto, D., Heinke, D., Humphreys, G. W., \& Blanco, M. J. (2005). Early, involuntary top-down guidance of attention from working memory. Journal of Experimental Psychology: Human Perception and Performance, 31, 248-261.

Sparks, D. L., \& Hartwitch-Young, R. (1989). The deep layers of the superior colliculus. In R. H. Wurtz \& M. E. Goldberd (Eds.), The neurobiology of saccadic eye movements (pp. 213255). Amsterdam: Elsevier.

Taylor, T. L. (2007). Inhibition of return for expected and unexpected targets. Acta Psychologica, 124, 257-273.

Taylor, T. L., \& Klein, R. M. (1998). On the cause and effects of inhibition of return. Psychonomic Bulletin \& Review, 5, 625-643.

Theeuwes, J., \& Godijn, R. (2004). Inhibition of return and oculomotor interference. Vision Research, 44, 1485-1492.
Theeuwes, J., Van der Sigchel, S., \& Olivers, C. N. L. (2006). Spatial working memory and inhibition of return. Psychonomic Bulletin \& Review, 13, 608-613.

Treisman, A., \& Gelade, G. (1980). A feature integration theory of attention. Cognitive Psychology, 12, 97-136.

Vivas, A. B., Humphreys, G. W., \& Fuentes, L. J. (2003). Inhibitory processing following damage to the parietal lobe. Neuropsychologia, 41, 1531-1540.

Vivas, A. B., Humphreys, G. W., \& Fuentes, L. J. (2006). Abnormal inhibition of return: A review and new data on patients with parietal lobe damage. Cognitive Neuropsychology, 23, 10491064.

Vivas, A. B., Humphreys, G. W., \& Fuentes, L. J. (2008). Objectbased inhibition of return in patients with parietal lobe damage. Neuropsychology, 22, 169-176.

Wolfe, J. M. (1994). Guided Search 2.0: A revised model of visual search. Psychonomic Bulletin \& Review, 1, 202-238.

Wolfe, J. M. (2007). Guided Search 4.0: Current progress with a model of visual search. In W. Gray (Ed.), Integrated models of cognitive systems (pp. 99-119). New York: Oxford University Press.

Woodman, G. F., \& Luck, S. J. (2007). Do the contents of visual working memory automatically influence attentional selection during visual search? Journal of Experimental Psychology: Human Perception and Performance, 33, 363-377.

Yantis, S., \& Jonides, J. (1984). Abrupt visual onsets and selective attention: Evidence from selective search. Journal of Experimental Psychology: Human Perception and Performance, 10, 601621. 